\title{
Sensor and Locomotion Concepts for Sewage Inspection and Repair Robots
}

\author{
H. Roth, K. Schilling \\ University of Applied Sciences Ravensburg-Weingarten \\ P.O.-Box 1261, D-88241 Weingarten \\ Germany \\ Tel: +49-751-501 627, Fax: +49-751-501654 \\ roth@fbe.fh-weingarten.de
}

\begin{abstract}
Sewerage is a very large and important infrastructure in water industry. Because around $20 \%$ of the municipal pipe network is estimated to be significantly damaged it is of high priority to inspect, maintain, and repair public sewers. During the last years mobile robots have been developed to inspect but also to repair the pipes. To navigate through the hostile environment, they are generally only equipped with video cameras and connected to the outside world via an umbilical cord which transmits the image information. The tethered robots are tele-operated from a remote service station placed at the end of a maintenance shaft. These pipe robots are commonly wheeled systcms especially adapted to withstand the adverse conditions of the waste pipe environment. In this paper the actual state of the art in the field of mobile robots for repair and inspection of sewerage is given and the possible improvements of the next generation of robots caused by developments of the sensors, the material, the tele-operation techniques and the locomotion principles are reported. This will allow the robots to increase their capabilities and to enlarge the application areas to pipes with smaller diameters and pipes in industrial plants.
\end{abstract}

Key words: Robotics, vision, sensors, tele-operation

\section{INTRODUCTION}

Sewerage is a very largc and important infrastructure in water industry. Because the municipal pipe network not only in Germany, but all over Europe is many years old, current estimates indicate that around $20 \%$ of the existing sewers suffer from significant damage and/or are leaking. This on the one side may contaminate the surrounding soil and ground water and on the other side ground water penetrates into the pipe and demands larger sewage works than necessary, producing high costs for the regional governments. Consequently, it is of high priority to inspect, maintain, and repair public sewers.

Because most of the pipes have a diameter between $200 \mathrm{~mm}$ and $600 \mathrm{~mm}$ they are not accessible for men. Therefore in earlier times the majority of repairs to pipes were carried out from above ground. If a defective pipe section was detected, a trench was dug to reach the pipe for repair. This approach is very costly and causes disruption if public areas such as streets are affected.

During the last years mobile robots have been developed to inspect but also to repair the pipes. To navigate through the hostile environment, they are generally only equipped with video cameras and connected to the outside world via an umbilical cord which transmits the image information. The tethered robots are tele-operated from a remote service station placed at the end of a maintenance shaft. These pipe robots are commonly wheeled systems especially adapted to withstand the adverse conditions of the waste pipe environment.

In the moment the next generation of mobile inspection and repair robots is under development. The main goals are

- to increase the robot's capabilities with respect to sensorics, tele-operation and locomotion,

- to enlarge the application areas offering the capability to inspect and repair pipes with smaller diameters up to $100 \mathrm{~mm}$ and pipes in industrial plants.

The main objectives of this paper are to describe the actual state of the art in the field of mobile robots for repair and inspection of sewerage and give an outlook to the capabilities of the next generation of robots, where the authors are working on. Due to the requirements forced by the hazardous environment and the dimensions of the pipes, the development is a challenge to the sensors, the material, the teleoperation techniques and the locomotion principles. 


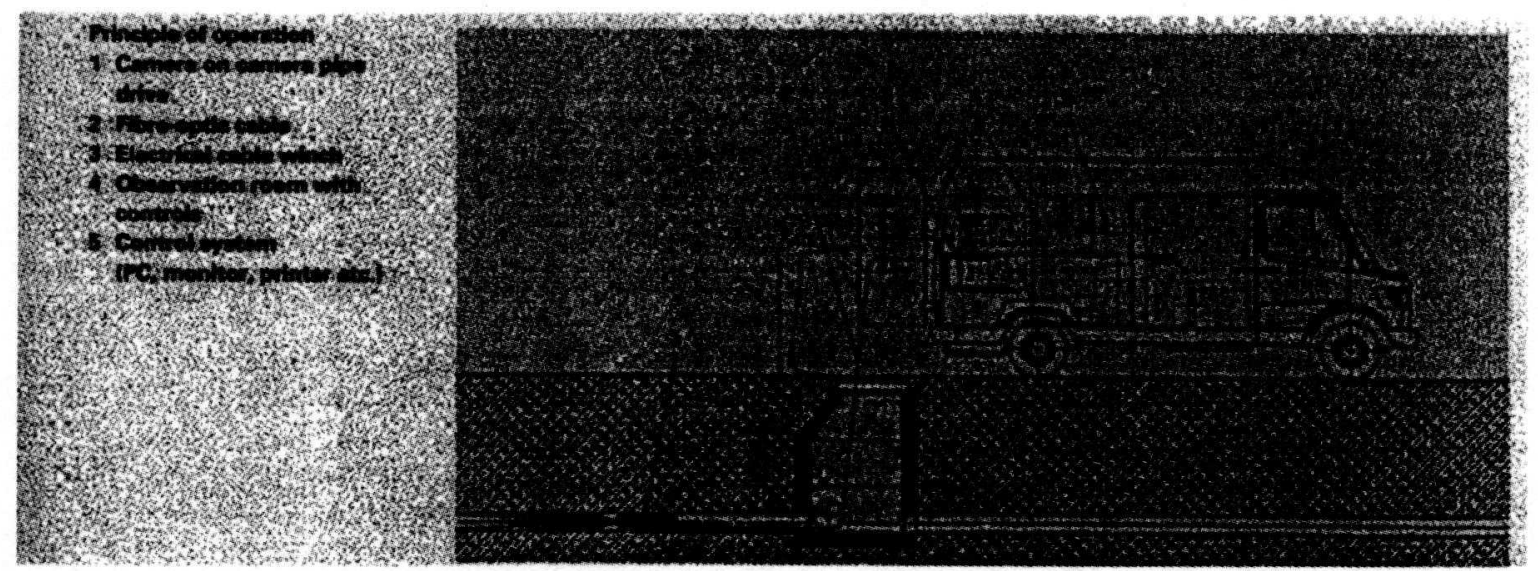

Figure 1: Typical equipment for waste water pipe inspection

\section{STATE OF THE ART}

The research and development of waste pipe robots is still in its infancy with limited examples of robots used for on site pipe repair. Due to the user needs for inspection and repair robots in pipe systems, there are several systems offered on the European market. Main emphasis is currently placed on inspection systems only. These tethered devices (for transmission of energy, data and concrete material for repair) are tele-operated by workers from the remote service station, placed at the end of a maintenance shaft (Figure 1). Problem areas for workers to navigate the robot through pipe systems concern poor or non-existing maps of the pipe net, mud deposits, running liquids, displaced pipe segments, causing gaps or steps, narrow pipe bendings or branch-offs with different diameters, requiring complex motions to pass by. Usually only the imaging system (camera and lighting equipment) supports the navigation tasks.

Repair is usually done by trenchless and commonly employed technology where the inside of the pipe is covered with a new coating. The "Sliplining" approach is one of the earliest forms of continuous structural lining where a new pipeline of smaller diameter is inserted into the defective pipe and the annulus is grouted. A modified variant of sliplining, called "Close Fit Lining", uses properties of PE (polyethelene) or PVC to temporarily reduce the diameter prior to inscrtion. The inserted tube is then expanded to form a tight fit against the wall of the original pipe, thus avoiding the need for annulus grouting as in conventional "Sliplining". Pipes showing only minor signs of deterioration can be repaired employing a technique called "Spray-on Lining". A high speed rotating head is winched through the pipe centrifugally depositing the lining material, such as cement mortar, quick setting epoxy resin or polyurethane material. Main problem areas are intersections of pipes. At these locations usually parts of the pipes are broken, the soil behind is washed away and lcakage is very high. Recent developments enable to repair this kind of damage by innovative repair functions, where the tele-operated robot injects special concrete to these leakage areas (Figure 2). Thus very cost efficiently pipes in the ground can be repaired at minimum disturbances, without digging holes into the ground.

Pipe robots are commonly wheeled systems especially adapted to withstand the adverse conditions of the waste pipe environment. For inspection and repair different types of robots are used. While the inspection robots usually are lightweight and small and often without own drives (they are drawn by cords), the repair robots, especially the milling cutters have to be very heavy and have their own drives. In this case slipping of the whecls is a common problem. It may occur due to running liquids (a typical water level in a sewer is 5\%), displaced pipe segments (causing gaps or steps), pipe bends and branch-offs. Further, a typical sewer system may consist of pipes from different matcrials. such as concrete, PVC, and clay, and this may also contribute to slipping. Further complications are obstacles frequently found in waste pipes; these include clogs, mud deposits, cracks, holes, connections, and roots grown into the pipes through cracks. For repair of these obstacles often milling cutters are necessary. In order to operate the milling cutter at the available very limited volume within the pipe, special mechanical devices have been designed.

\section{ACTUAL DEVELOPMENTS}

It is the main objective of the actual research, to increase the capabilities of existing pipe robots for inspection and repair by innovative use of microsensorics, new manipulators and tele-operation features to develop a pipe robot according to important application needs. In actual research projects, the authors are working in the development of a new generation of mobile inspection and repair robots. Departing from already existing repair robot products innovative improvements are investigated to increase the robot's capabilities as well as the application areas 

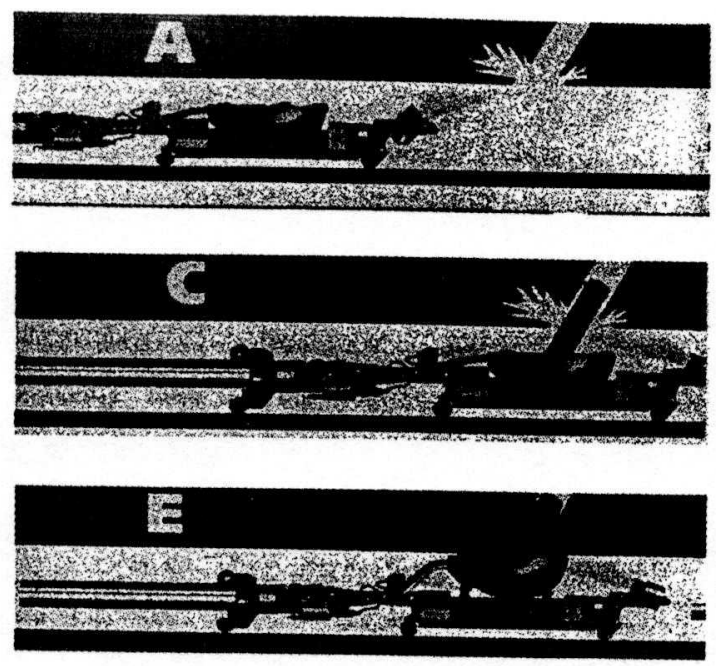
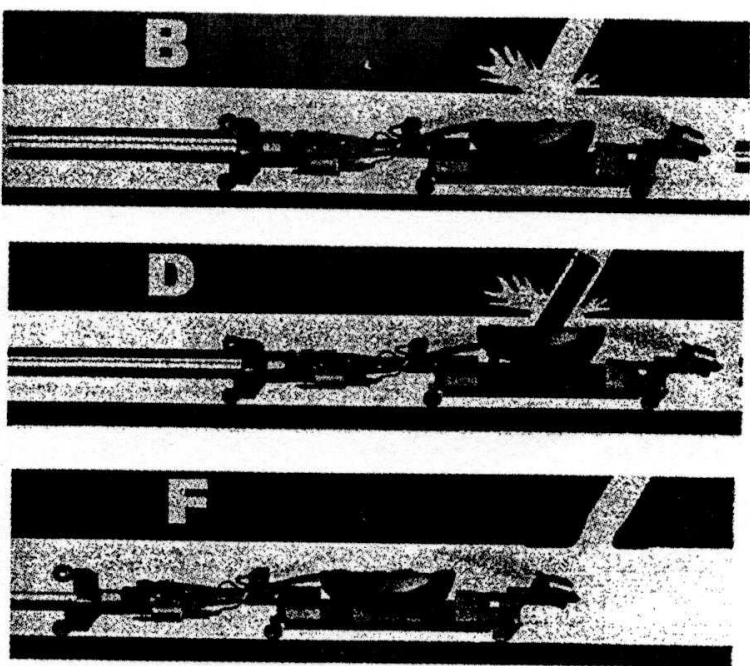

Figure 2: Repair process at damaged pipe intersections

- by the use of additional sensors (like contact sensors, range sensors, gyros, inclinometers) and micro-sensors to navigate and accurately position the robot for inspection and repair, as well as to monitor the actual status of the repair process,

- by the use of sensors (video imaging, laser profiling system and ultra sonic sensors) to acquire images of the inner pipe face and on-line analysis of the acquired data to detect and classify obstacles, cracks and leakages,

- by pipe failure recognition, based on data base and expert system techniques,

- by implementing a robust, but very flexible and powerful milling cutter to prepare the leakage area for the repair,

- by adapting the robot for navigation and uninterrupted operations in curved pipes of small diameter beyond a distance of $100 \mathrm{~m}$,

- by the use of remote tele-operation supported by intelligent (neural-network-based and fuzzybased) navigation strategies to steer the robot in the uncertain pipe environment.

- by remote access to the service station via modem link to a central station in order to provide technical assistance in robot operations by specialists.

In order to expand application areas especially for repair purposes, it is desirable to offer an innovative, very flexible arm with a milling cutter to prepare the repair area appropriately before injecting the concrete. To operate this milling cutter, appropriate sensor information on the repair area, the robot arm's position and the forces occurring at the milling cutter have to be provided to the worker. Due to the limited space available, standard sensors and cameras can not be used in this context. Here the application of new micro-sensors is in test. As a consequence of the unclean environment robust sensors are necessary and advanced post-processing methods sensor data by filtering [4] and for sensor data fusion have to be employed. Despite the interesting potential, modern smart sensor technologies seem not to have been applied so far in this context.

Related micro-sensors for contact detection (minibumpers, force sensors), range determination (infrared, ultrasonic sensors), navigation (odometry, gyros, inclinometers, accclerometers), vision and imaging (miniature CCD-cameras integrated with processing unit on the same chip) have been developed and applied by the authors for mobile robot applications in space exploration [1], [2] and in industrial transport tasks [3]. In actual research projects the authors are adapting these developments to the needs of pipe robots.

New locomotion concepts combining hydraulic, electronic and optical components driving the robot enables unprecedent industrial performance of the robot in small diameter pipes or in long pipes, in parallel with low costs, when considering the reduced diameter, complex shapes, reach and ability to navigate without interrupting the service. There are developments in using intelligent decision systems for pipe maintenance and operations, based on neurofuzzy pattern recognition techniques and inspection database integrating images, numerical data and expert knowledge.

These improvements will significantly increase the application spectrum of pipe robots. The available experience from earlier micro-robot projects (for hazardous environments, for space exploration on Mars and Moon [1]) encourages the authors that the challenging objectives in this area are realistic and achievable.

\section{CONCLUSIONS AND OUTLOOK}

In this paper an overview have been given about the existing robot technology for inspection and repair of waste water pipes. In addition the actual state of the research areas are reported in order to 
increase the robot's capabilities with respect to sensorics, tele-operation and locomotion, and to enlarge the application areas offering the capability to inspect and repair pipes with smaller diameters up to $100 \mathrm{~mm}$ and pipes in industrial plants.

Until the conference first results of the design of a prototype can be reported, demonstrating the increasing possibilities for navigation and repair, if support is given by different smart sensors. The mechanical design and the locomotion principle will in addition allow this robot to inspect pipes with small diameters.

\section{REFERENCES}

[1] K. Schilling, H. Roth: Teleoperations Concept and Sensor System Design of the Mars Rover MIDD, Proceedings of the 3rd IFAC Symposium on Intelligent Autonomous Vehicles, Madrid, Spain, 1998.

[2] K. Schilling, R. Lieb, H. Roth: Control and Operations Procedures for Short-Range Planetary Micro-Rovers, Proceedings "International Conference on Mobile Planetary Robots", Santa Monica, USA, January 1997.

[3] K. Schilling, R. Lieb, H. Roth: Indoor Navigation of Mobile Robots Based on Natural Landmarks, Proceedings "3rd IFAC Symposium on Intelligent Components and Instruments for Control Applications", Annecy, France 1997. p.527-530.

[4] R. Doyle, H. Roth, Das Kalman Filter, in: Tränkler / Obcrmeier (eds.) Sensortechnik Handbuch für Praxis und Wirtschaft, Springer Verlag 1998. 